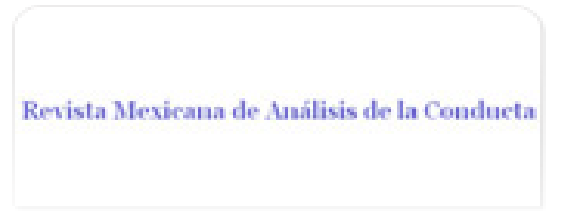

Revista Mexicana de Análisis de la Conducta ISSN: 0185-4534

editora@rmac-mx.org

Sociedad Mexicana de Análisis de la Conducta México

López, Christian; Bruner, Carlos A.

Efectos de Tiempo Relativo Sobre una Discriminación Basada en el Reforzamiento Diferencial de la Respuesta que Produce el Agua en una Situación de Beber Inducido por el Programa Revista Mexicana de Análisis de la Conducta, vol. 35, núm. 2, diciembre, 2009, pp. 39-56

Sociedad Mexicana de Análisis de la Conducta

Guadalajara, México

Disponible en: http://www.redalyc.org/articulo.oa?id=59311902004

Cómo citar el artículo

- Número completo

- Más información del artículo

Página de la revista en redalyc.org

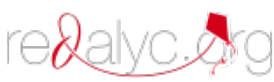

Sistema de Información Científica

Red de Revistas Científicas de América Latina, el Caribe, España y Portugal Proyecto académico sin fines de lucro, desarrollado bajo la iniciativa de acceso abierto 


\title{
Efectos de Tiempo Relativo Sobre una Discriminación Basada en el Reforzamiento Diferencial de la Respuesta que Produce el Agua en una Situación de Beber Inducido por el Programa
}

\author{
Relative Time Effects on a Discrimination Based \\ on the Differential Reinforcement of the Water Producing Response \\ in a Schedule-Induced-Drinking-Procedure \\ CHRISTIAN LÓPEZ Y CARLOS A. BRUNER ${ }^{1}$ \\ UNIVERSIDAD NACIONAL AUTÓNOMA DE MÉXICO
}

Resumen

La mayoría de los intentos previos por condicionar el beber inducido por el programa (BIP) a estímulos arbitrarios involucraron procedimientos de condicionamiento clásico, con resultados mixtos. Considerando la hipótesis de que el BIP se reduce al condicionamiento operante de la respuesta que produce el agua, en un experimento previo se estableció el control del estímulo sobre el BIP mediante un programa múltiple de reforzamiento con agua. Para probar que la discriminación operante en el caso del BIP es sensible al parámetro de la duración de los componentes de un programa múltiple como cualquier operante, se expuso a ratas privadas de comida a un programa de tiempo al azar $60 \mathrm{~s}$ por comida y concurrentemente a programas de reforza-

1. El presente estudio formó parte de la tesis doctoral del primer autor, cuyos estudios fueron apoyados mediante la beca 181559 del CONACYT y la beca para estudios de doctorado de la DGEP. Los autores contribuyeron en la misma proporción en la elaboración del presente estudio y agradecen los valiosos comentarios de tres revisores anónimos que ayudaron a mejorar el escrito. Dirigir correspondencia a: Laboratorio de Condicionamiento Operante, Facultad de Psicología, UNAM; Ave. Universidad 3004, Cd. Universitaria, México D. F. 04510. México (e-mail: cbruner@ servidor.unam.mx ochlg78@yahoo.com).

Recibido: 30 de abril, 2009. Revisado: 24 de mayo, 2009. Aceptado: 25 de septiembre, 2009 
miento con agua mixtos y un múltiple. En ambos programas, el componente de reforzamiento duró $32 \mathrm{~s}$ y se reforzó con agua el palanqueo mediante un programa de intervalo al azar $6 \mathrm{~s}$. El componente de extinción duró 16 o 256 $\mathrm{s}$, para cada tres ratas. Se estableció una discriminación durante el programa múltiple cuando el componente de extinción duró $256 \mathrm{~s}$, pero no cuando el componente de extinción duró $16 \mathrm{~s}$. Este resultado muestra que el BIP se puede sujetar a una discriminación operante que sigue el principio de tiempo relativo.

Palabras clave: beber inducido por el programa, reforzamiento con agua, discriminación operante, efectos de tiempo relativo, ratas.

\begin{abstract}
Most previous attempts to condition schedule-induced drinking (SID) to arbitrary stimuli have involved classical-conditioning procedures, with mixed results. Considering the hypothesis that SID reduces to the operant reinforcement of the water-producing response, in a previous experiment the control of a stimulus on SID was established using a multiple water reinforcement schedule. To show that the operant discrimination of SID is sensitive to the duration of the extinction component of a multiple schedule as any other operant, food deprived rats were exposed to a 60 -s random time food schedule and concurrently to mixed and multiple water-reinforcement schedules. On both schedules, reinforcement components lasted $32 \mathrm{~s}$ and rats lever-pressed for water on a 6-s random interval schedule. Extinction components lasted either 16 or $256 \mathrm{~s}$, for each three rats. During the multiple schedule an operant discrimination was formed when the extinction component lasted $256 \mathrm{~s}$, but not when it lasted 16 $\mathrm{s}$. This result show that SID can be subjected to an operant discrimination and that such discrimination follows the principle of relative time.
\end{abstract}

Key words: schedule-induced drinking, water reinforcement, operant discrimination, relative time effects, rats.

El beber inducido por el programa (BIP) consiste en el consumo sustancial de agua de ratas privadas de comida, el cual ocurre al exponerlas a un programa de entrega de comida (Falk, 1961). Para tratar de explicar el BIP conforme a los principios establecidos en la teoría de la conducta, se intentó probar que el BIP constituía un caso de condicionamiento operante en el que la comida reforzaba accidentalmente el consumo de agua (Stein, 1964). Está explicación se descartó cuando se observó en varios estudios que programas con una demora reiniciable entre el consumo de agua y la entrega de comida no impedía el desarrollo del BIP (e.g., Falk, 1964). 
En otro intento por integrar al BIP a los principios establecidos en la teoría de la conducta, se propuso que en los procedimientos de BIP el consumo de agua era una respuesta incondicionada provocada por la entrega comida (e.g., Rosenblith, 1970). Para probar está explicación en diversos estudios se intentó determinar si un estímulo arbitrario apareado con la entrega de comida se podía condicionar Pavlovianamente para evocar el consumo de agua en un procedimiento de BIP. Se encontró que el control del estímulo sobre el consumo de agua fue inconsistente entre diferentes estudios (cf. Wetherington, 1982). En algunos estudios se reportó que las presentaciones del estímulo sin la comida controlaron un aumento del consumo de agua (e.g., Corfield-Sumner, Blackman \& Stainer, 1977), mientras que en otros estudios se reportó que las presentaciones del estímulo sin la comida controlaron una disminución del consumo de agua (e.g., Allen, Porter \& Arazie, 1975).

López y Bruner (2003) dedujeron que la evidencia contradictoria respecto al control del estímulo sobre el BIP podía deberse a que se utilizaron procedimientos en los que se varió inconsistentemente la duración del intervalo entre comidas, el número de estímulos presentados y la posición de estos estímulos dentro del intervalo entre comidas. A diferencia de los estudios anteriores en los que se intentó determinar el control de un estímulo arbitrario sobre el BIP, López y Bruner añadieron un estímulo y variaron sistemáticamente su ubicación temporal dentro de un intervalo entre comidas constante, entre grupos (Experimento 1) o entre fases con los mismos sujetos (Experimento 2), con la finalidad de observar los efectos del estímulo sobre el patrón de consumo de agua controlado por la entrega de la comida. López y Bruner encontraron que el estímulo modificó el patrón temporal de consumo de agua para cada sujeto, aunque el efecto del estímulo sobre el patrón de consumo de agua fue diferente entre sujetos. Mediante el estudio de López y Bruner se determinó que utilizando procedimientos de condicionamiento Pavloviano únicamente se puede establecer un control supersticioso del estímulo sobre el consumo de agua.

Los resultados inconsistentes acerca del control del estímulo neutral sobre el BIP, así como los fracasos por reducir al BIP a un caso de condicionamiento supersticioso, se utilizaron como parte de la evidencia a favor de la idea de que el BIP pertenece a una tercera clase de conducta diferente de la conducta operante y respondiente (Falk, 1977). Proponer una nueva categoría de conducta para explicar un fenómeno aparentemente anómalo representa un serio problema para el análisis experimental de la conducta debido a que le resta generalidad a los principios establecidos en la teoría de la conducta (Cabrer, Daza \& Ribes, 1975; Skinner, 1938).

Para tratar de explicar el BIP conforme a los principios establecidos en la teoría de la conducta, Bruner y Ávila (2002) propusieron que la conducta que produce el agua, como lamer un tubo, era reforzada directamente con la 
obtención de agua. Los investigadores sugirieron que el procedimiento para producir BIP implicaba establecer inadvertidamente una privación de agua en la caja habitación como resultado de privar de comida a los sujetos y que la entrega de comida a intervalos de tiempo durante las sesiones experimentales restablecía el consumo de agua. Para probar que el BIP podía ser controlado mediante procedimientos de condicionamiento operante, Bruner y Ávila reforzaron intermitentemente con una gota de agua las presiones a una palanca conforme a programas de intervalo fijo (IF 64, 32, 16 y $8 \mathrm{~s}$, en condiciones sucesivas) y concurrentemente entregaron comida conforme a un programa de tiempo al azar (TA) $60 \mathrm{~s}$. Encontraron que la respuesta de presión a la palanca por agua se estableció y mantuvo en las tres ratas con un diferente valor del IF para cada rata. Bruner y Ávila demostraron que es posible el condicionamiento operante de la conducta de beber mediante el reforzamiento intermitente con agua de la conducta que produce el agua en una situación de BIP, i.e. en ratas privadas de comida y durante la entrega de comida a intervalos de tiempo.

Dada la demostración de que el BIP constituye un caso de condicionamiento operante en el cual el agua es el verdadero reforzador de la respuesta que la produce (Bruner \& Ávila, 2002), en un estudio realizado por López y Bruner (2007) se demostró que es posible establecer el control de un estímulo neutral sobre el BIP si tan solo se utilizan procedimientos de discriminación operante en los que los estímulos estén correlacionados con el agua y no con la comida como en los estudios anteriores. En su estudio López y Bruner expusieron a tres ratas privadas de comida a programas mixtos y múltiples de reforzamiento con agua. En componentes de $32 \mathrm{~s}$, las presiones a una palanca produjeron agua conforme a un programa de reforzamiento de intervalo al azar (IA) $6 \mathrm{~s}$. En componentes de $64 \mathrm{~s}$ las presiones a la palanca no produjeron reforzamiento con agua. Concurrentemente, durante todo el estudio se entregó comida conforme a un programa de tiempo al azar (TA) 60 s. Para las tres ratas, la tasa de respuesta fue similar en los dos componentes cuando se mantuvieron en efecto los programas mixtos. También para las tres ratas, la tasa de respuesta fue más alta en los componentes de reforzamiento con agua que en los componentes de extinción cuando se mantuvieron en efecto los programas múltiples. Los autores mostraron que el reforzamiento diferencial de la respuesta que produce el agua en presencia del estímulo es una condición crucial para establecer el control del estímulo sobre el beber. En contraste con los estudios en los que se utilizaron procedimientos de condicionamiento Pavloviano para establecer el control de un estímulo sobre el BIP, el estudio de López y Bruner constituyó la primera demostración confiable del control de un estímulo previamente neutral sobre el BIP utilizando procedimientos de discriminación operante. 
En el área de discriminación operante está bien establecido que la discriminación entre estímulos está sujeta a parámetros que regulan la función de los estímulos discriminativos (Nevin, 1973; Skinner, 1938). En el caso de la discriminación operante establecida en una situación de BIP reportada por López y Bruner (2007), también se esperaría que fuera sensible a los parámetros conocidos de una discriminación operante. Uno de los parámetros más importantes en el establecimiento de una discriminación operante es variar la duración de los componentes de un programa múltiple de reforzamiento. Los efectos de está manipulación se han observado sobre la amplitud de la separación entre las tasas de respuesta en los componentes de reforzamiento y de extinción de los programas múltiples (Todorov, 1972; Williams, 1983). Por ejemplo, Wilton y Clements (1971) entrenaron a palomas a picar una tecla por comida bajo un programa de reforzamiento de IV $60 \mathrm{~s}$. Posteriormente expusieron a los sujetos a un programa múltiple IV $60 \mathrm{~s}$ EXT. La duración del componente de reforzamiento se mantuvo constante en $20 \mathrm{~min}$, mientras que en diferentes sesiones se alargó la duración del componente de EXT de 10 $\mathrm{s}$ a $120 \mathrm{~min}$. En cada sesión se presentó únicamente un componente de extinción y un componente de reforzamiento. Los autores reportaron que la tasa de carrera en el componente de reforzamiento fue una función creciente de aumentar la duración del componente de extinción. Los resultados reportados en varios estudios sobre discriminación operante en los que se replicaron los hallazgos reportados por Wilton y Clements al variar la duración del componente de extinción manteniendo constante la duración del componente de reforzamiento en programas múltiples de reforzamiento, pueden considerarse como ejemplos del principio de tiempo relativo (De Rose, 1986; Ettinger \& Staddon, 1982; Hinson \& Staddon, 1981; McSweeney \& Melville, 1993). Se conoce como principio de tiempo relativo al aumento en la fuerza de la respuesta durante un periodo de reforzamiento señalado de duración constante, que ocurre como resultado de aumentar la duración del intervalo entre presentaciones sucesivas del periodo de reforzamiento señalado (Williams, 1980, 1998). El principio de tiempo relativo es un fenómeno robusto y sus efectos sobre la conducta se han probado bajo diferentes procedimientos de condicionamiento, entre los que se encuentran procedimientos de automoldeamiento y automantenimiento (Bruner, 1981; Terrace, Gibbon, Farrell \& Baldrock, 1975), de supresión condicionada (e.g., Stein, Sidman \& Brady, 1958), de igualación a la muestra (e.g., Schaal, Odum \& Shahan, 2000), de reforzamiento condicionado (e.g., Fantino, 1969), de reforzamiento demorado (e.g., Ruiz, Bruner \& Balderrama, 2007) y de discriminación (e.g., Terrace, 1966).

Considerando que López y Bruner (2007) demostraron que la respuesta que produce el agua se puede someter al control discriminativo de los estímulos de un programa múltiple en un procedimiento de BIP, resulta importante mostrar que el BIP es sensible al parámetro de la duración los componentes 
de reforzamiento y extinción de un programa múltiple de reforzamiento como cualquier conducta arbitraria en un procedimiento operante (e.g., Wilton \& Clements, 1971). En el presente estudio se probó si, en comparación con las tasas de respuesta observadas durante el programa de reforzamiento múltiple del estudio de López y Bruner, es posible aumentar o disminuir la diferencia entre las tasas de respuesta en los componentes de reforzamiento y de extinción, únicamente como resultado de alargar o acortar los componentes de extinción respecto a la duración de $64 \mathrm{~s}$ del componente de extinción utilizado por López y Bruner. Para garantizar que durante las sesiones experimentales se mantuvieran presentes las condiciones del BIP, se utilizaron ratas privadas de comida y se entregó comida durante las sesiones.

\section{MÉTODO}

\section{Sujetos}

Se utilizaron seis ratas Wistar macho de tres meses de edad, experimentalmente ingenuas. Se controló la cantidad de alimento entregado a las ratas en las cajas habitación para mantenerlas al $80 \%$ de su peso ad libitum. Las ratas tuvieron acceso continuo al agua en las cajas habitación.

\section{Aparatos}

Se utilizaron tres cajas experimentales Med Associates Inc. (ENV-007). En cada caja se instaló una palanca (ENV-110RM) sensible a una fuerza de 0.15 $\mathrm{N}$, que se ubicó al centro del panel frontal y a $7 \mathrm{~cm}$ del piso de las cajas. A la izquierda de la palanca y a $1 \mathrm{~cm}$ del piso de las cajas se instaló un recipiente para el agua (ENV-200R3M) conectado mediante una manguera a una válvula solenoide (ENV-201A) colocada en la parte posterior del panel frontal. La válvula entregó gotas de agua de $0.1 \mathrm{~mL}$. A la derecha de la palanca y a $1 \mathrm{~cm}$ del piso de las cajas se instaló un recipiente para la comida (ENV-200R1AM) conectado mediante una manguera a un dispensador de bolitas de comida (ENV-203IR) ubicado en la parte posterior del panel frontal. Se utilizaron bolitas de comida de $25 \mathrm{mg}$, que se fabricaron remoldeando polvo de comida para ratas (Harland Teklad). En el panel frontal también se colocó un sonalert (ENV-223AM) ubicado $25 \mathrm{~cm}$ por arriba de la palanca y dos focos de $28 \mathrm{~V}$ (ENV-221M), uno ubicado $6 \mathrm{~cm}$ por arriba de la palanca y el otro ubicado 11 $\mathrm{cm}$ por arriba del recipiente para la comida. El sonalert produjo un tono de $2.9 \mathrm{kHz}$ y $75 \mathrm{~dB}$. Al centro del panel posterior y a $25.5 \mathrm{~cm}$ del piso se instaló un foco de $28 \mathrm{~V}$ (ENV-215M) que proporcionó iluminación continua durante las sesiones. En el panel posterior también se colocó un generador de ruido 
blanco (ANL-914A) ubicado a la derecha del foco y a $19.5 \mathrm{~cm}$ del piso de las cajas, el cual ayudó a enmascarar sonidos ajenos al experimento. Se introdujo a cada caja experimental dentro de una caja sonoamortiguada (ENV-018) equipada con un ventilador (ENV-025FAC) que facilitó la circulación del aire. Desde una habitación contigua a la habitación en la cual se ubicaron las cajas experimentales, se controlaron y registraron los eventos experimentales mediante una computadora con software Med-PC versión IV, acoplada a una interfase Med Associates Inc.(SG-503).

\section{Procedimiento}

Con el propósito de entrenar la respuesta de presión a la palanca, se expuso a las seis ratas a 20 sesiones en las que se reforzaron las presiones a la palanca conforme a un programa de reforzamiento con agua de IA $6 \mathrm{~s}(t=$ $2 \mathrm{~s}, p=.33)$ y concurrentemente se presentó un programa de TA $60 \mathrm{~s}$ por comida ( $t=6 \mathrm{~s}, p=.1)$. Cada sesión duró 1 hora o 160 reforzadores de agua entregados, lo que ocurriera primero.

Se asignó a las ratas a dos grupos de tres ratas cada uno. Se igualaron entre sí a ambos grupos de ratas conforme a la tasa media de respuesta de las ratas durante las últimas cinco sesiones de exposición al IA $6 \mathrm{~s}$. En tres condiciones sucesivas, se presentó a los dos grupos de ratas un programa de reforzamiento con agua mixto IA $6 \mathrm{~s}$ EXT, un programa de reforzamiento con agua múltiple IA 6 s EXT y un programa de reforzamiento con agua mixto IA 6 s EXT, respectivamente. Durante estas tres condiciones el componente de reforzamiento duró $32 \mathrm{~s}$ para los dos grupos de ratas. El componente de extinción duró $16 \mathrm{~s}$ para un grupo de ratas y $256 \mathrm{~s}$ para el otro grupo de ratas. En la condición con el programa de reforzamiento múltiple se señaló a los componentes de reforzamiento con el encendido y apagado cada segundo del sonalert y de los dos focos y a los componentes de extinción con el encendido constante del sonalert y los dos focos. Cada condición duró 30 sesiones y cada sesión terminó después de que se presentaron 30 componentes de reforzamiento y 30 componentes de extinción. Los componentes de reforzamiento y de extinción se alternaron al azar con la restricción de que no ocurrieran en sucesión más de dos componentes de un mismo tipo. Durante los programas mixtos y el múltiple se presentó concurrentemente el programa de TA $60 \mathrm{~s}$ por comida.

La duración del componente de reforzamiento y los valores de los programas de entrega de comida de TA y de reforzamiento con agua de IA, se eligieron con el propósito de que el procedimiento del presente estudio fuera similar al del estudio de López y Bruner (2007). Las duraciones del componente de extinción de 16 y 256 s utilizadas en el presente estudio se calcula- 
ron dividiendo entre 4 o multiplicando por 4 respectivamente, el valor de $64 \mathrm{~s}$ de la duración del componente de extinción utilizado por López y Bruner.

\section{RESULTADOS}

En la literatura sobre discriminación operante, la tasa de respuesta ha sido una de las principales variables dependientes con la cual se han medido los efectos de un procedimiento de discriminación sobre la conducta (Dinsmoor, 1995). En la Figuras 1a y $1 \mathrm{~b}$ se presenta para cada sujeto la tasa media de presiones a la palanca en cada sesión, para las condiciones con los programas mixtos y el múltiple. Se puede observar en las figuras que durante los programas mixtos la tasa de respuesta durante el componente de reforzamiento fue similar a la tasa de respuesta durante el componente de extinción para las seis ratas. También se observó que la tasa de respuesta de las ratas que fueron expuestas al componente de extinción de $16 \mathrm{~s}$ fue más alta que la tasa de respuesta de las ratas que fueron expuestas al componente de extinción de $256 \mathrm{~s}$. Cuando se presentó el programa múltiple la tasa de respuesta de los sujetos expuestos a la duración del componente de extinción de $16 \mathrm{~s}$ permaneció sin cambios respecto a la condición anterior. En contraste, para los sujetos expuestos a la duración del componente de extinción de $256 \mathrm{~s}$ se encontró que la tasa de respuesta durante el componente de reforzamiento aumentó respecto a la condición previa y que la tasa durante el componente de extinción siguió siendo baja al igual que en la condición previa. 


\section{Componente de EXT de $16 \mathrm{~s}$}

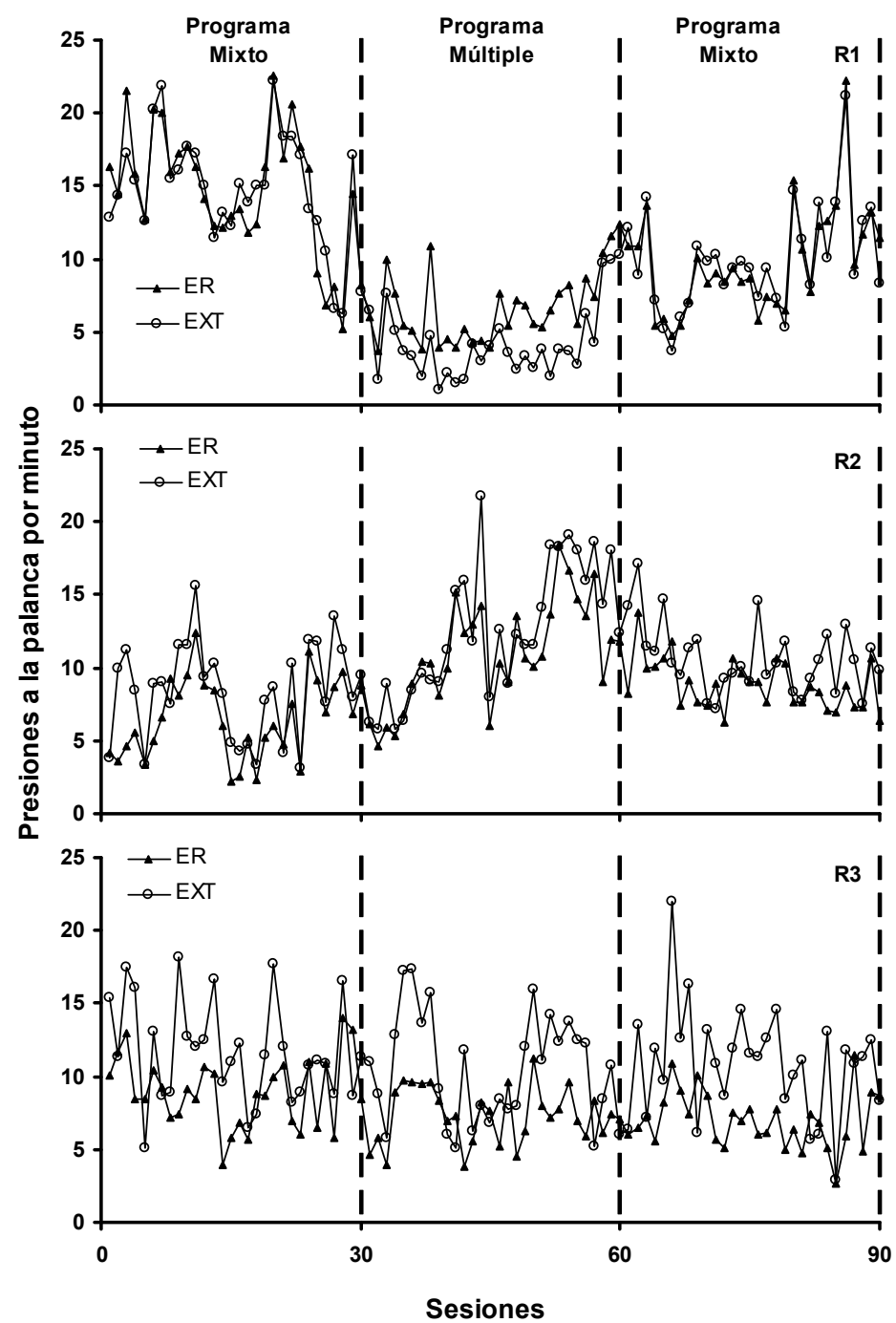

Figura 1a. Tasa media de respuesta en cada sesión en los componentes de reforzamiento (ER) y de extinción (EXT), durante los programas mixtos y el múltiple, para los sujetos expuestos al componente de extinción de $16 \mathrm{~s}$. 


\section{Componente de EXT de $256 \mathrm{~s}$}

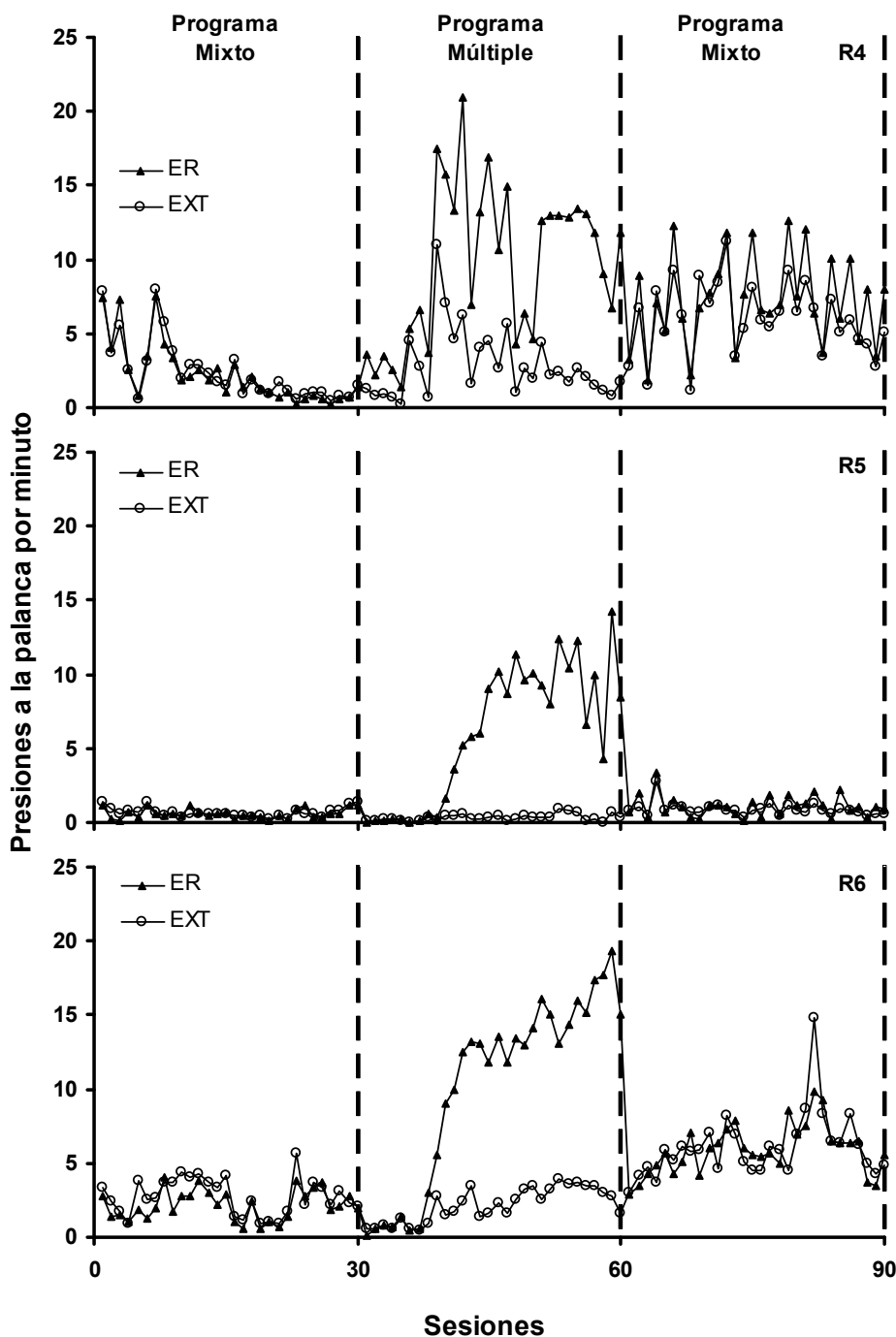

Figura 1b. Tasa media de respuesta en cada sesión en los componentes de reforzamiento (ER) y de extinción (EXT), durante los programas mixtos y el múltiple, para los sujetos expuestos al componente de extinción de 256 s. 
El índice de discriminación del estímulo es un análisis reportado frecuentemente en estudios en los que se utilizan programas múltiples debido a que permite observar el control de los estímulos sobre la tasa de respuesta (Nevin, 1973). En las Figuras 2a y $2 b$ se presenta el índice de discriminación en función del paso de las sesiones de exposición a los programas mixtos y el múltiple. El índice de discriminación se calculó dividiendo la tasa de respuesta obtenida durante el componente de reforzamiento entre la suma de la tasa de respuesta obtenida durante el componente de reforzamiento y de extinción (Dinsmoor, 1952). Durante las condiciones con los programas mixtos se encontró que el índice de discriminación para las ratas expuestas al componente de extinción de $16 \mathrm{~s}$ fue similar al índice de discriminación para las ratas expuestas al componente de extinción de 256s, que fue de aproximadamente 0.5 . En la condición con el programa múltiple se observó en dos de las tres ratas expuestas al componente de extinción de $16 \mathrm{~s}$, que el índice de discriminación permaneció sin cambios respecto a la condición previa. Para la Rata 1 expuesta al componente de extinción de $16 \mathrm{~s}$ se observó durante el programa múltiple un ligero aumento del índice de discriminación. Durante el programa múltiple se encontró para las ratas con el componente de extinción de $256 \mathrm{~s}$ un aumento en el índice de discriminación respecto a la condición con el programa mixto. 


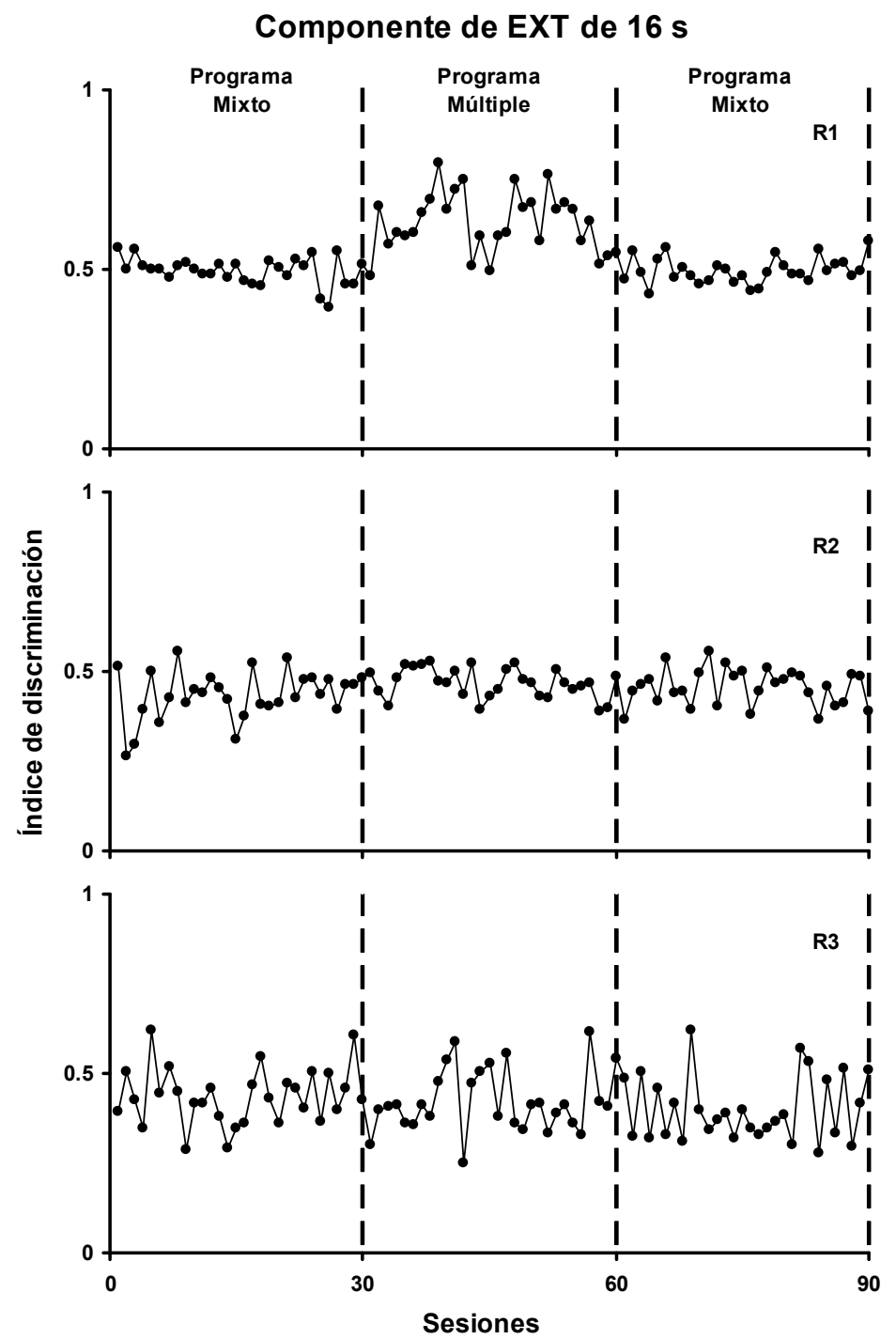

Figura 2a. Índice de discriminación del estímulo en función del paso de las sesiones de exposición a los programas mixtos y el múltiple, para los sujetos expuestos al componente de extinción de $16 \mathrm{~s}$. 


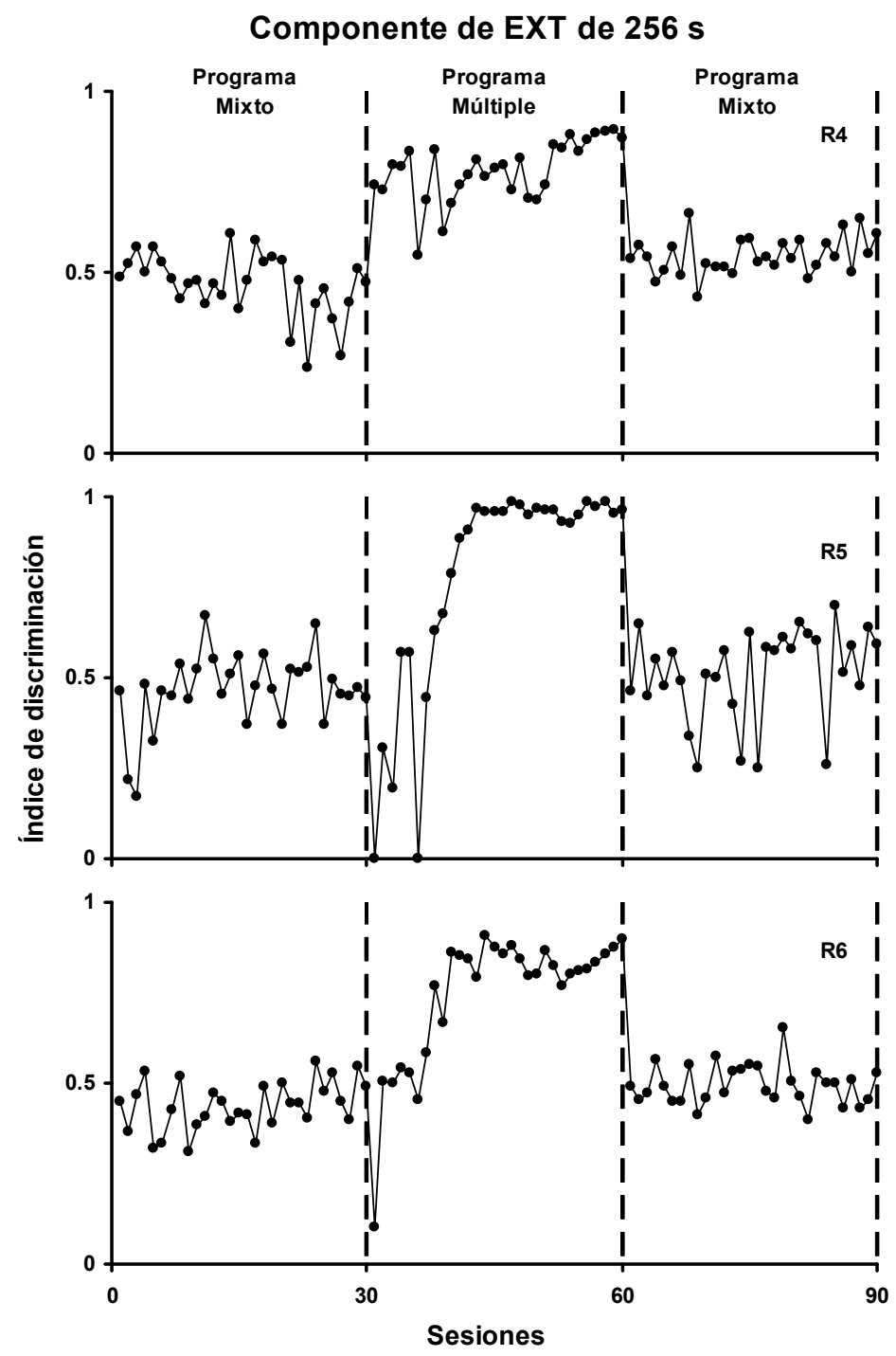

Figura $2 b$. Índice de discriminación del estímulo en función del paso de las sesiones de exposición a los programas mixtos y el múltiple, para los sujetos expuestos al componente de extinción de $256 \mathrm{~s}$. 
Con el propósito de comparar el efecto de la duración del componente de extinción del programa múltiple de reforzamiento sobre el índice de discriminación de los sujetos del presente estudio con los tres sujetos utilizados en el estudio de López y Bruner (2007), en la Figura 3 se presenta el promedio del índice de discriminación durante las últimas cinco sesiones con el programa de reforzamiento múltiple, para los tres sujetos del estudio de López y Bruner y para los seis sujetos del presente estudio. Se encontró un aumento del índice de discriminación de los estímulos conforme aumentó la duración de los componentes de extinción del programa múltiple de reforzamiento.

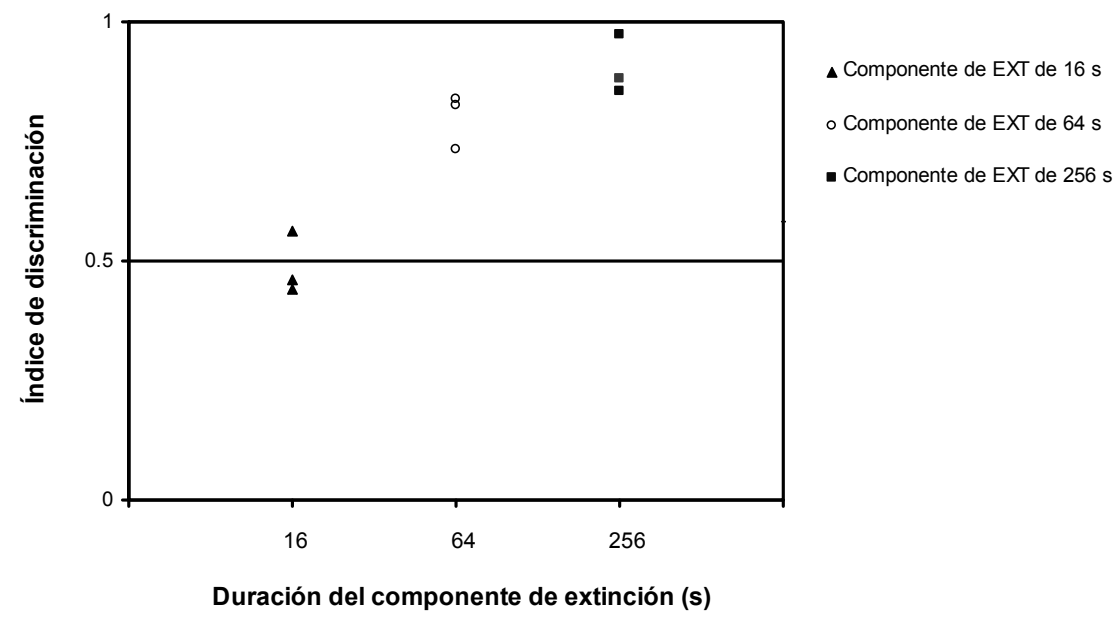

Figura 3. Índice de discriminación promedio durante las últimas cinco sesiones con el programa múltiple, para los tres sujetos del estudio de López y Bruner (2007) con componente de extinción de 64 s y para los seis sujetos del presente estudio con los componentes de extinción de 16 o $256 \mathrm{~s}$.

\section{DISCUSIÓN}

El propósito del presente estudio fue probar si en comparación con las tasas de respuesta observadas durante el programa de reforzamiento múltiple utilizado en el estudio de López y Bruner (2007), era posible aumentar o disminuir la diferencia entre las tasas de respuesta en los componentes de reforzamiento y de extinción como resultado de alargar o acortar la duración del componente 
de extinción respecto a la duración del componente de extinción de $64 \mathrm{~s}$ utilizado por López y Bruner, en una situación de BIP. Al comparar los resultados de López y Bruner con el presente estudio se encontró que el índice de discriminación de los estímulos aumentó en función de los aumentos en la duración del componente de extinción del programa de reforzamiento múltiple. Este hallazgo confirma que durante el establecimiento de una discriminación, la tasa de la respuesta que produce el agua en una situación de BIP es sensible a la manipulación de una variable típica de los estudios de discriminación operante con programas múltiples al igual que cualquier otra conducta arbitraria controlada por un procedimiento de discriminación operante (cf., Williams, 1983).

En los intentos anteriores por establecer el control de un estímulo previamente neutral sobre el BIP se utilizaron procedimientos de condicionamiento Pavloviano (Wetherington, 1982). Este tipo de procedimientos no permitieron establecer claramente el control del estímulo sobre el consumo de agua, ya que mientras que en algunos estudios el estímulo neutral controló un aumento del consumo de agua, en otros estudios con procedimientos similares el estímulo controló una disminución del consumo de agua (e.g., Allen, Porter \& Arazie, 1975; Corfield-Sumner, Blackman \& Stainer, 1977). Los hallazgos inconsistentes sobre el control del estímulo neutral sobre el BIP se utilizaron como parte de la evidencia en favor de la propuesta de que el BIP pertenece a una tercera clase de conducta (Falk, 1977). En contraste con los hallazgos de los estudios en los que se utilizaron procedimientos de condicionamiento Pavloviano para determinar el control de un estímulo previamente neutral sobre el BIP, en el presente estudio se estableció el control de estímulos previamente neutrales sobre el BIP, reforzado diferencialmente con agua a la respuesta que produce el agua en presencia de dos diferentes estímulos. Este hallazgo muestra que no es necesario explicar al BIP como parte de una tercera clase de conducta y en cambio, fortalece la idea de que el BIP es un caso de condicionamiento operante (Bruner \& Ávila, 2002).

En el presente estudio es sorprendente que a pesar de que los sujetos no se encontraban explícitamente privados del reforzador durante las sesiones experimentales (i.e., el agua), se observaron resultados similares a los resultados reportados en los estudios tradicionales sobre discriminación operante en los que se investigaron los efectos de la duración de los componentes de un programa múltiple de reforzamiento utilizando a sujetos privados del estímulo que posteriormente se utiliza como reforzador durante las sesiones experimentales (e.g., Wilton \& Clements, 1971). En estudios recientes sobre el BIP se mostró que el origen de las propiedades reforzantes del agua durante las sesiones de BIP depende del momento en el que se entrega la comida tanto en la caja habitación, como en la cámara experimental, dada la estrecha relación que existe entre el consumo de comida y de agua (Bruner \& Ávila, 2002; Roca \& Bruner, 2007). Se ha observado que al privar de comi- 
da a las ratas en su caja habitación su consumo de agua disminuye, lo cual significa que el investigador produce inadvertidamente una privación indirecta de agua al privar a la rata de comida en los procedimientos de BIP. Una vez que los sujetos comienzan a recibir comida en la cámara experimental el consumo de agua que dejó de ocurrir en la caja habitación se restablece durante las sesiones experimentales. En el presente estudio el origen de las propiedades reforzantes del agua durante las sesiones experimentales probablemente se explica mediante este mismo mecanismo, lo cual contribuye a aclarar por qué los resultados del presente estudio son similares a los resultados de los estudios sobre discriminación operante en los que se priva a los sujetos del estímulo que se utiliza como reforzador, a pesar de que no se privó directamente de agua a las ratas.

En los estudios en los que se reportaron efectos de tiempo relativo se emplearon diversos procedimientos de condicionamiento (Williams, 1980). Por ejemplo, se reportaron efectos de tiempo relativo en estudios sobre automoldeamiento con palomas, en los que se investigó el control del intervalo entre ensayos sobre la velocidad de adquisición del picoteo a la tecla de respuesta. En estos estudios, cada ensayo consistió en presentar en la tecla de respuesta un estímulo visual breve de duración constante seguido de la entrega de comida. Durante el intervalo entre ensayos la tecla de respuesta permaneció obscura. Se encontró que el picoteo a la tecla de respuesta se estableció con mayor rapidez conforme aumentó el intervalo entre ensayos para diferentes grupos de palomas (e.g., Bruner, 1981; Gibbon, Baldrock, Locurto, Gold \& Terrace, 1977; Terrace, Gibbon, Farrell \& Baldrock, 1975). También se reportaron efectos de tiempo relativo en diferentes estudios sobre discriminación operante con programas múltiples (e.g., De Rose, 1986; Ettinger \& Staddon, 1982; Hinson \& Staddon, 1981; McSweeney \& Melville, 1993; Wilton \& Clements, 1971). Por ejemplo, De Rose investigó el efecto de variar la duración del componente de extinción de un programa múltiple IF por comida EXT, sobre las presiones a una palanca de ratas privadas de comida. En diferentes condiciones se utilizaron tres duraciones del componente de reforzamiento. Manteniendo constante la duración del componente de reforzamiento, en cada condición se probaron diferentes duraciones del componente de extinción. De Rose encontró que la tasa de respuesta durante el componente de reforzamiento fue una función directa de la duración del componente de extinción. También encontró que la pausa en el responder al inicio de cada componente de reforzamiento se acortó conforme se alargó la duración del componente de extinción. El autor concluyo que el cambio en la duración del componente en el que se suspendió el reforzamiento determinó la magnitud de las diferencias entre las tasas de respuesta en los componentes del programa múltiple. Los hallazgos del presente estudio permitieron demostrar que al igual que otros fenómenos reportados con diferentes 
procedimientos de condicionamiento, la discriminación de la respuesta que produce el agua en una situación de BIP también está sujeta a los principios de tiempo relativo, lo cual muestra a su vez que el BIP es una instancia de conducta condicionada y no de una tercera clase de conducta.

\section{REFERENCIAS}

Allen, J. D., Porter, J. H., \& Arazie, R. (1975). Schedule-induced drinking as a function of percentage reinforcement. Journal of the Experimental Analysis of Behavior, 23, 223-232.

Bruner, C. A. (1981)._. The effect of cycle length, interstimulus interval and probability of reinforcement in autoshaping/automaintenance. Disertación Doctoral, City University of New York, 1981. Ann Arbor, Michigan: University Microfilms, No. 81-2057.

Bruner, C. A., \& Ávila, R. (2002). Adquisición y mantenimiento del palanqueo en ratas sin privación explícita del reforzador. Revista Mexicana de Análisis de la Conducta, 28, 107-130.

Cabrer, F., Daza, B. C., \& Ribes, E. (1975) Teoría de la Conducta: ¿Nuevos conceptos o nuevos parámetros?. Revista Mexicana de Análisis de la Conducta, 1, 191-212.

Corfield-Sumner, P. K, Blackman, D. E., \& Stainer, G. (1977). Polyidipsia induced in rats by second-order schedules of reinforcement. Journal of the Experimental Analysis of Behavior, 27, 265-273.

De Rose, J. C. C. (1986). Behavioral contrast in fixed-interval components: Effects of extinction-component duration. Journal of the Experimental Analysis of Behavior, $45,175-188$.

Dinsmoor, J. A. (1952). The effect of hunger on discriminated responding. Journal of Abnormal and Social Psychology, 47, 67-72.

Ettinger, R. H., \& Staddon, J. E. R. (1982). Behavioral competition, component duration and multiple-schedule contrast. Behaviour Analysis Letters, 2, 31-38.

Falk, J. L. (1961). Production of polydipsia in normal rats by an intermittent food schedule. Science, 133, 195-196.

Falk, J. L. (1964). Studies on schedule-induced polydipsia. En M. J. Wayner (Ed.), Thirst: First International Symposium on thirst in the regulation of body water (pp. 95-116). New York: Pergamon Press.

Falk, J. L. (1977). The origin and functions of adjunctive behavior. Animal Learning \& Behavior, 5, 325-335.

Fantino, E. (1969). Choice and rate of reinforcement. Journal of the Experimental Analysis of Behavior, 12, 723-730.

Gibbon, J., Baldock, M. D., Locurto, C., Gold, L., \& Terrace, H. S. (1977). Trial and intertrial durations in autoshaping. Journal of Experimental Psychology: Animal Behavior Processes, 3, 264-284.

Hinson, J. M., \& Staddon, J. E. R. (1981). Some temporal properties of local contrast. Behaviour Analysis Letters, 1, 275-281. 
López, C., \& Bruner, C. A. (2003). Efectos del intervalo estímulo-comida sobre la polidipsia en ratas. Revista Mexicana de Análisis de la Conducta, 29, 193-211.

López, C. G., \& Bruner, C. A. (2007). La formación de una discriminación operante en una situación de beber inducido por el programa. Revista Mexicana de Análisis de la Conducta, 33, 99-117.

McSweeney, F. K., \& Melville, C. L. (1993). Behavioral contrast for key pecking as a function of component duration when only one component varies. Journal of the Experimental Analysis of Behavior, 60, 331-343.

Nevin, J. A. (1973). Stimulus control. In J. A. Nevin (Ed.), The study of behavior (pp. 115-152). Glenview, IL: Scott, Foresman.

Roca, A., \& Bruner C. A. (2007). La polidipsia psicógena se reduce a un artefacto de procedimiento. Revista Mexicana de Psicología, 24, 209-218.

Rosenblith, J. Z. (1970). Polydipsia induced in the rat by a second-order schedule. Journal of the Experimental Analysis of Behavior, 12, 139-144.

Ruiz, J. A., Bruner, C. A., \& Balderrama, D. M. (2007). Efecto de tiempo relativo en demoras de reforzamiento señaladas y no señaladas. Revista Mexicana de Análisis de la Conducta, 33, 119-138.

Schaal D. W, Odum A. L, \& Shahan T. A. (2000). Pigeons may not remember the stimuli that reinforced their recent behavior. Journal of the Experimental Analysis of Behavior, 73, 125-139.

Skinner, B. F. (1938). The behavior of organisms. New York: Appleton-CenturyCrofts.

Stein, L. (1964). Excessive drinking in the rat: superstition or thirst?. Journal of Comparative and Physiological Psychology, 58, 237-242.

Stein, L., Sidman, M., \& Brady, J. V. (1958). Some effects of two temporal variables on conditioned suppression. Journal of the Experimental Analysis of Behavior, 1, 153-162.

Terrace, H. S. (1966). Stimulus control. In W. K. Honig (Ed.), Operant Behavior. New York: Appleton-Century-Crofts.

Terrace, H. S., Gibbon, J., Farrell, L., \& Baldock, M. D. (1975). Temporal factors influencing the acquisition of an autoshaped response. Animal Learning \& Behavior, 3, 53-62.

Todorov, J. C. (1972). Component duration and relative response rates in multiple schedules. Journal of the Experimental Analysis of Behavior, 17, 45-49.

Wetherington, C. L. (1982). Is adjuntive behavior a third class of behavior?. Neuroscience and Biobehavioral Reviews, 6, 329-350.

Wilton, R. N., \& Clements, R. O. (1971). Behavioral contrast as a function of the duration of an immediately preceding period of extinction. Journal of the Experimental Analysis of Behavior, 16, 425-428.

Williams, B. A. (1980). Contrast, signaled reinforcement, and the relative law of effect. American Journal of Psychology, 93, 617-629.

Williams, B. A. (1983). Another look at contrast in multiple schedules. Journal of the Experimental Analysis of Behavior, 39, 345-384.

Williams, B. A. (1998). Relative time and delay of reinforcement. Learning and Motivation, $29,236-248$. 